MATHEMATICS OF COMPUTATION

Volume 74, Number 251, Pages 1457-1470

S 0025-5718(04)01701-6

Article electronically published on July 21, 2004

\title{
REAL ZEROS OF DEDEKIND ZETA FUNCTIONS OF REAL QUADRATIC FIELDS
}

\author{
KOK SENG CHUA
}

\begin{abstract}
Let $\chi$ be a primitive, real and even Dirichlet character with conductor $q$, and let $s$ be a positive real number. An old result of H. Davenport is that the cycle sums $S_{\nu}(s, \chi)=\sum_{n=\nu q+1}^{(\nu+1) q-1} \frac{\chi(n)}{n^{s}}, \nu=0,1,2, \ldots$, are all positive at $s=1$, and this has the immediate important consequence of the positivity of $L(1, \chi)$. We extend Davenport's idea to show that in fact for $\nu \geq 1, S_{\nu}(s, \chi)>0$ for all $s$ with $1 / 2 \leq s \leq 1$ so that one can deduce the positivity of $L(s, \chi)$ by the nonnegativity of a finite $\operatorname{sum} \sum_{\nu=0}^{t} S_{\nu}(s, \chi)$ for any $t \geq 0$. A simple algorithm then allows us to prove numerically that $L(s, \chi)$ has no positive real zero for a conductor $q$ up to 200,000 , extending the previous record of 986 due to Rosser more than 50 years ago. We also derive various estimates explicit in $q$ of the $S_{\nu}(s, \chi)$ as well as the shifted cycle sums $T_{\nu}(s, \chi):=\sum_{n=\nu q+\lfloor q / 2\rfloor+1}^{(\nu+1) q+\lfloor q / 2\rfloor} \frac{\chi(n)}{n^{s}}$ considered previously by Leu and Li for $s=1$. These explicit estimates are all rather tight and may have independent interests.
\end{abstract}

\section{INTRODUCTION}

Let $\chi$ be a primitive real Dirichlet character. It is a fundamentally difficult and profound problem to show that the Dirichlet $L$-function $L(s, \chi):=\sum_{n=1}^{\infty} \frac{\chi(n)}{n^{s}}$ is nonvanishing for real $s>0$. In the case of odd character $\chi(-1)=-1$, which corresponds to the imaginary quadratic field, the $L$ series can be related to Epstein zeta functions of positive definite binary quadratic forms which have rapidly converging expansions (see [1 and [10]) and in $1968 \mathrm{M}$. Low [6] devised a method which allowed him to show that $L(s, \chi)>0$ for $s>0$ for a negative conductor up to $-593,000$. Recently this has been corrected and extended by M. Watkins [13] to $-300,000,000$. Apparently, only recently in [2] has the positivity of $L(s, \chi)$ throughout $s \in[1 / 2,1]$ been proven for an infinite number of primitive Dirichlet $L$ functions by Conrey and Soundararajan. They proved that this holds for at least $20 \%$ of the primitive odd characters of conductor $-8 d$ for positive odd square-free $d$.

On the other hand, little seems to have been done in the case of even character $\chi(-1)=1$. Apparently the best published method is that of Rosser, who showed, more than 50 years ago in [7] and in an unpublished work that $L(s, \chi)$ has no real positive zero for a positive conductor up to 986 .

In this note we extend an old idea of $\mathrm{H}$. Davenport 3 and give a simple computational method for showing the nonvanishing of $L(s, \chi)$ for real positive $s$ for even real primitive characters. Our key observation is that the partial cycle sums

Received by the editor November 15, 2003 and, in revised form, February 21, 2004.

2000 Mathematics Subject Classification. Primary 11M20; Secondary 11M06.

Key words and phrases. Real zeros of $L$ functions, Dirichlet series, primitive characters. 
$S_{\nu}(s, \chi)=\sum_{n=\nu q+1}^{(\nu+1) q-1} \frac{\chi(n)}{n^{s}}$ are positive for $1 / 2 \leq s \leq 1$ and $\nu=1,2,3, \ldots$ This extends Davenport's observation from $s=1$. It implies that if we can show that an exact finite sum $\sum_{\nu=0}^{t} S_{\nu}(s, \chi)$ is nonnegative for some $t \geq 0$, then we can deduce $L(s, \chi)>0$ for the fixed $s$. A simple idea using the mean value theorem allows us to extend this to an algorithm for verifying the nonvanishing of $L(s, \chi)$ for $1 / 2 \leq s \leq 1$ (see ALGO 1 in subsection 2.2). By the functional equation [4 and the easy fact that $L(s, \chi) \neq 0$ for $\operatorname{Re}(s)>1$, we are able to verify numerically the following result:

Theorem 1.1. Let $\chi$ be a real and even primitive Dirichlet character with conductor $q$. Then $L(s, \chi)$ has no real positive zero for $q \leq 200,000$.

Since $\zeta_{\mathbf{Q}(\sqrt{q})}(s)=\zeta(s) L(s, \chi)$, this implies that the corresponding Dedekind zeta functions have no positive real zero. The reader with more computing power should be able to extend the range of $q$ in Theorem 1.1 using our method. In subsection 2.1, we give the key result: Theorem 2.1, which is the extension of Davenport's result but we also give estimates for the partial cycle sum explicit in $q$. The algorithm used for verifying positivity and a discussion of some possible extensions is given in subsection 2.2. Proofs of our results and the estimates are given in Section 3 while Section 4 contains further estimates extending results of Leu and Li [5] who generalized Davenport's idea to shifted cycle sums. Theorem 4.1 is an analogue of Theorem 2.1. Finally in Section 5 we give the results of our numerical computations.

\section{MAIN IDEAS AND RESUlts}

2.1. An extension of a result of Davenport. Let $\chi$ be a primitive Dirichlet character $\bmod q$. We define for any real $s>0$,

$$
S_{\nu}(s, \chi):=\sum_{n=\nu q+1}^{(\nu+1) q} \frac{\chi(n)}{n^{s}}, \quad \nu=0,1,2, \ldots
$$

In 1949, in response to a question of Erdös, H. Davenport proved, "with little difficulty" 3] that if $\chi$ is real and even, $S_{\nu}(1, \chi)>0$ for all $\nu \geq 0$. By continuity, one expects that this should extend to a small neighborhood (depending on $q$ ) to the left of $s=1$ but one does not expect to be able to prove this easily because of obvious implications to the question of Siegel's zero 9 , 11. However one can easily extends Davenport's arguments to prove the following.

Theorem 2.1. If $\chi$ is real, primitive and even, and $1 / 2 \leq s \leq 1$, then we have

$$
S_{\nu}(s, \chi)>0, \text { for } \nu=1,2,3, \ldots
$$

More precisely, we have the following explicit lower bounds for $1 / 2 \leq s \leq 1$ :

$$
\begin{aligned}
& S_{1}(s, \chi)>\frac{s}{40 q^{s-1 / 2}}\left(1-\frac{5}{2^{s+3}}\right) \\
& S_{\nu}(s, \chi)>\frac{s}{40 q^{s-1 / 2}}\left(\frac{1}{\nu^{1+s}}-\frac{1}{(\nu+1)^{1+s}}\right), \text { for } \nu=2,3, \ldots
\end{aligned}
$$

In view of (2.2), we define for $t=0,1,2,3, \ldots$,

$$
L_{t}(s, \chi):=\sum_{\nu=0}^{t} S_{\nu}(s, \chi) \text {, }
$$


which increases monotonically and converges to $L(s, \chi)$. By $(2.2)$ it suffices for us to show that $L_{t}(s, \chi) \geq 0$ for any $t \geq 0$ to conclude that $L(s, \chi)>0$. By the Extended Riemann Hypothesis, such a finite $t$ must exist. Indeed in most cases, it suffices to choose $t=0$. In fact $L_{t}(s, \chi)$ converges rapidly to $L(s, \chi)$ for any primitive characters (not necessarily real or even). Let us set

$$
L(s, \chi)=L_{t}(s, \chi)+E_{t+1}(s, \chi) .
$$

Then we have the following explicit bound for the error:

Theorem 2.2. Let $\chi$ be any primitive character $\bmod q$ and $s>0$. Then we have

$$
\begin{aligned}
& \left|E_{t+1}(s, \chi)\right| \leq \frac{1}{q^{s-1 / 2}}\left(\frac{s}{12(t+1)^{s+1}}+\frac{s(s+1)(s+2)}{720(t+1)^{s+3}}\right) \quad \text { if } \chi \text { is even, } \\
& \left|E_{t+1}(s, \chi)\right| \leq \frac{1}{q^{s-1 / 2}}\left(\frac{|L(1, \chi)|}{\pi(t+1)^{s}}+\frac{s(s+1) \zeta(3)}{4 \pi^{3}(t+1)^{s+2}}\right), \text { if } \chi \text { is odd. }
\end{aligned}
$$

Let us say that a primitive modulus $q$ for a real even character is exceptional for $s, s \in[0,1]$ if $S_{0}(s, \chi)<0$. Davenport's result is that there is no exceptional $q$ for $s=1$ and we expect this to remain true for $s$ near 1 . Numerically, we have found that up to $1,000,000$, there is no exceptional $q$ for $s=0.555$ or 0.56 and there are only two at 14,693 and 788,933 at $s=0.55$. Indeed it may be the case that exceptional $q$ does not exist for $s>0.555$. Indeed for all the $q$ values up to 200,000 that we consider which are exceptional for $s=1 / 2$, we have verified numerically that $S_{0}(s, \chi)$ is increasing on $[1 / 2,1]$ and the unique value $1 / 2<\alpha(q)<1$ where $S_{0}(\alpha(q), \chi)=0$ is given to 5 decimal places in the last column of Table 1 below. For fixed $s>1 / 2$, we expect that all sufficiently large $q$ should be nonexceptional in view of the factor $\frac{1}{q^{1 / 2-s}}$ in (2.6). Even for the worst case $s=1 / 2$, the first exceptional $q$ occurs at $q=13340$ and there are only 218 exceptional $q \leq 1,000,000$ and among these $L_{1}(1 / 2, \chi)>0$ for 174 of them. Up to one million, the largest least $t$ required for $L_{t}(s, \chi)$ to become positive is 8 for $q=925,097$. Table 1 in Section 5 below contains a list of all exceptional $q \leq 200,000$ at 0.5 and the smallest $t$ value in each case.

2.2. A method for verifying $L(s, \chi)>0$ for $0<s<1$. Theorem 2.1 gives us a way to prove that $L(s, \chi)>0$ at a particular point $s$ by evaluating an exact finite sum. However the following simple observation allows us to extend it to a numerical method for verifying that $L(s, \chi)>0$ in an interval. By the functional equation [4], it suffices for us to check this for $1 / 2 \leq s \leq 1$ for us to deduce that it has no real zero.

Lemma 2.3. Let $f(s) \in C^{1}[a, b]$ and assume that $\left|f^{\prime}(s)\right| \leq g(s)$ for $s \in[a, b]$ and a function $g(s)$ which is positive and decreasing on $[a, b]$. Then from $f\left(s_{0}\right)>0$ for some $s_{0} \in(a, b)$, we can deduce $f(s)>0$ for $s \in\left[s_{0}, s_{0}+\frac{f\left(s_{0}\right)}{g\left(s_{0}\right)}\right)$.

Proof. Clearly we may assume $f(s) \leq f\left(s_{0}\right)$. We then have

$$
f\left(s_{0}\right)-f(s) \leq \int_{s_{0}}^{s}\left|f^{\prime}(t)\right| d t \leq \int_{s_{0}}^{s} g(t) d t \leq g\left(s_{0}\right)\left(s-s_{0}\right) .
$$

So we have $f(s) \geq f\left(s_{0}\right)-g\left(s_{0}\right)\left(s-s_{0}\right)>0$ for $s_{0} \leq s<s_{0}+\frac{f\left(s_{0}\right)}{g\left(s_{0}\right)}$. 


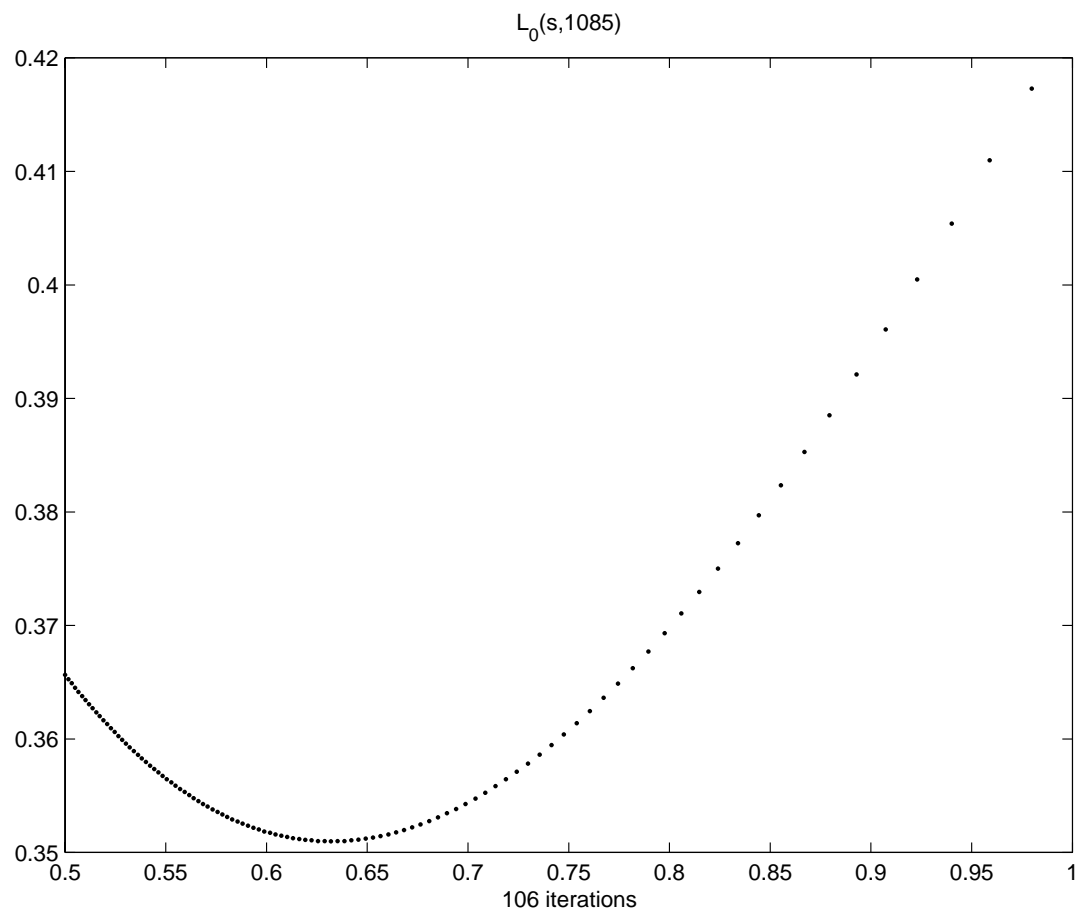

FiguRE 1. $\mathrm{q}=1085$.

We may apply Lemma 2.3 to $f(s)=L_{t}(s, \chi)$ since

$$
\left|L_{t}^{\prime}(s, \chi)\right| \leq \sum_{(n, q)=1, n=2}^{(t+1) q-1} \frac{\log n}{n^{s}}:=g(s)
$$

is clearly decreasing. We start with $s_{0}=1 / 2$ and pick a $t$ (for example the least $t)$ such that $L_{t}(1 / 2, \chi)>0$. Such a $t$ must exist if $L(1 / 2, \chi)>0$ which in turn is widely believed to be true and K. Soundararajan [12] has shown that at least $7 / 8$ of the quadratic fields have $L(1 / 2, \chi) \neq 0$. Computationally, M. Rubinstein 8 ] has computed $L(1 / 2, \chi)$ for $q$ up to $100,000,000$ to 15 decimal places, and they are all positive.

We then iteratively define the new point $s_{i+1}:=s_{i}+L_{t}\left(s_{i}, \chi\right) / g\left(s_{i}\right)$ and check the positivity of $L_{t}\left(s_{i+1}, \chi\right)$. When $s_{i+1} \geq 1$, we have verified the nonexistence of a real zero. In practice, we allow a small positive tolerance $\epsilon>0$ and the algorithm pseudocode is given below.

\section{ALGO 1}

Find a $t$ such that $L_{t}(1 / 2, \chi)>\epsilon$ and set $s=1 / 2$;

while $\left(s<1\right.$ and $\left.L_{t}(s, \chi)>\epsilon\right)$ do

$$
s=s+L_{t}(s, \chi) / \sum_{(n, q)=1, n=2}^{(t+1) q-1} \frac{\log n}{n^{s}} ;
$$

if $s>1$ then NO POSITIVE REAL ZERO 


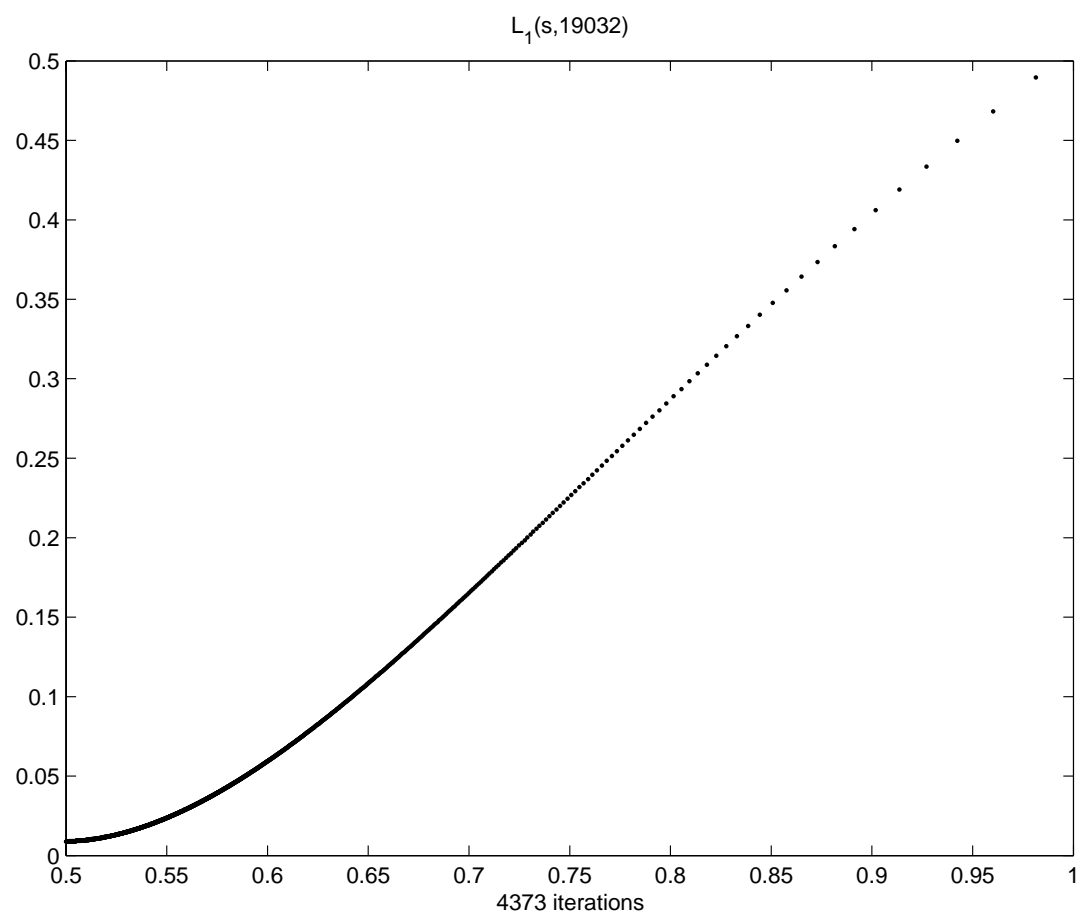

FiguRe 2. $\mathrm{q}=19032$.

In this way we obtain Theorem 1.1. The progress of the algorithms is best illustrated in the plots of $L_{0}(s, \chi)$ for $q=1085$ requiring 106 iterations in Figure 1 and $L_{1}(s, \chi)$ for $q=19032$ in Figure 2 requiring 4373 iterations for $\epsilon=10^{-5}$. The $t$ values used are the least with $L_{t}(1 / 2, \chi)>0$. These are the simplest cases. More iterations are needed usually (see Table 1). More details of the numerical computations will be given in Section 5 .

ALGO 1 has the defect that the program will fail if we encounter an $s$ with $1 / 2<s<1$ and $L_{t}(s, \chi)<\epsilon$ though $L_{t}(s, \chi)$ may be positive throughout (this does not occur in the range of $q$ that we computed above). One way to get around this is to note that the condition " $L_{t}(s, \chi) \geq 0 \Rightarrow L(s, \chi)>0$ " is pointwise so that we may choose different $t$ at different $s$. The obvious thing to do is to pick the least $t$ such that $L_{t}(s, \chi)>\epsilon$ for each $s$, which incurs no additional computational cost at each point evaluation since $L_{t}(s, \chi)$ is computed as $L_{t}(s, \chi)=L_{t-1}(s, \chi)+S_{t}(s, \chi)$ but we found experimentally that the pointwise computational saving is far outweighed by the larger number of iterations needed for using smaller $t$. The program will now work whenever $L(s, \chi)>\epsilon$ throughout $[1 / 2,1]$ but it may still be necessary to pick a sufficiently small $\epsilon$. However if we set $\alpha_{t}(s)$ to be the right-hand side of (2.6) and pick for each $s$ the least $t$ such that $L_{t}(s, \chi)>\alpha_{t}(s)$, the program is guaranteed to succeed as long as $L(s, \chi)>0$ in $[1 / 2,1]$. Note that such a $t$ must exist if $L(s, \chi)>0$ since $\alpha_{t}(s) \rightarrow 0$ in $t$ and $L_{t}(s, \chi)$ is eventually positive. It also has the advantage of ensuring that $L_{t}(s, \chi)>L(s, \chi) / 2$ so that it is more efficient because the number of iterations is much less than picking the least $t$ making $L_{t}(s, \chi)>0$. However the value of $t$ chosen will be 0 most of the time, and we find experimentally 
that the naive ALGO 1 with a fixed $t$ greater than the minimum necessary actually performed better.

\section{Proof of Main Results}

3.1. A Fourier expansion. In this section, we will prove our main estimates in Theorems 2.1 and 2.2 essentially following Davenport's argument extended to $s<1$. We consider for any real $s>0$ and $\nu=1,2,3, \ldots$ the expansion of

$$
f(x)=\frac{1}{(\nu+x)^{s}} \sim \frac{a_{0}}{2}+\sum_{m=1}^{\infty}\left(a_{m} \cos 2 \pi m x+b_{m} \sin 2 \pi m x\right), \quad 0<x<1,
$$

in a Fourier series of period 1.

The coefficients are

$$
a_{m}=2 \int_{0}^{1} \frac{\cos 2 \pi m x}{(\nu+x)^{s}} d x, \quad b_{m}=2 \int_{0}^{1} \frac{\sin 2 \pi m x}{(\nu+x)^{s}} d x .
$$

Integrating (3.1) by parts gives for each $k \geq 2$,

$$
a_{m}=\sum_{j=1}^{k}(-1)^{j-1} \frac{F_{2 j}}{m^{2 j}}+(-1)^{k} \frac{2 s(s+1) \cdots(s+2 k-1)}{(2 \pi m)^{2 k}} \int_{0}^{1} \frac{\cos 2 \pi m x}{(\nu+x)^{s+2 k}} d x
$$

$$
b_{m}=\sum_{j=1}^{k}(-1)^{j-1} \frac{F_{2 j-1}}{m^{2 j-1}}+(-1)^{k} \frac{2 s(s+1) \cdots(s+2 k-2)}{(2 \pi m)^{2 k-1}} \int_{0}^{1} \frac{\sin 2 \pi m x}{(\nu+x)^{s+2 k-1}} d x,
$$

where

$$
\begin{aligned}
& F_{1}=\frac{1}{\pi}\left\{\frac{1}{\nu^{s}}-\frac{1}{(\nu+1)^{s}}\right\} \\
& F_{j}=\frac{2 s(s+1) \cdots(s+j-2)}{(2 \pi)^{j}}\left\{\frac{1}{\nu^{s+j-1}}-\frac{1}{(\nu+1)^{s+j-1}}\right\}, \quad j \geq 2 .
\end{aligned}
$$

Lemma 3.1. For $k \geq 2$, we have

$$
\begin{aligned}
& a_{m}=\sum_{j=1}^{k-1}(-1)^{j-1} \frac{F_{2 j}}{m^{2 j}}+(-1)^{k-1} \theta_{m, k} \frac{F_{2 k}}{m^{2 k}}, \\
& b_{m}=\sum_{j=1}^{k-1}(-1)^{j-1} \frac{F_{2 j-1}}{m^{2 j-1}}+(-1)^{k-1} \phi_{m, k} \frac{F_{2 k-1}}{m^{2 k-1}},
\end{aligned}
$$

where $0<\theta_{m, k}<1,0<\phi_{m, k}<1$.

Proof. Since clearly we have

$$
\begin{aligned}
0<I_{m, 2 k}:= & \int_{0}^{1} \frac{\cos 2 \pi m x}{(\nu+x)^{s+2 k}} \\
& <\int_{0}^{1} \frac{d x}{(\nu+x)^{s+2 k}}=\frac{1}{s+2 k-1}\left\{\frac{1}{\nu^{s+2 k-1}}-\frac{1}{(\nu+1)^{s+2 k-1}}\right\},
\end{aligned}
$$

we may write $I_{m, 2 k}=\frac{1-\theta_{m, k}}{s+2 k-1}\left\{\frac{1}{\nu^{s+2 k-1}}-\frac{1}{(\nu+1)^{s+2 k-1}}\right\}$ for some $\theta_{m, k} \in(0,1)$. Equation (3.6) follows from (3.2). 
3.2. Proof of Theorem $\mathbf{2 . 2}$ for arbitrary primitive character. We first derive general estimates which hold for any primitive character $\chi$ (not necessary real or even) which may be of some independent interest. First we have (see [4, p. 68])

$$
\tau_{m}(\chi)=\sum_{n=1}^{q-1} \chi(n) e^{2 \pi i m n / q}=\bar{\chi}(m) \tau(\chi),
$$

where the Gauss sum $\tau(\chi):=\tau_{1}(\chi)$ satisfies $\tau(\chi) \tau(\bar{\chi})=\chi(-1) q$. It follows that

$$
\sum_{n=1}^{q-1} \chi(n) \cos 2 \pi m n / q=\bar{\chi}(m) \tau(\chi), \quad \sum_{n=1}^{q-1} \chi(n) \sin 2 \pi m n / q=0
$$

if $\chi$ is even, and

$$
\sum_{n=1}^{q-1} \chi(n) \cos 2 \pi m n / q=0, \quad \sum_{n=1}^{q-1} \chi(n) \sin 2 \pi m n / q=-i \bar{\chi}(m) \tau(\chi)
$$

if $\chi$ is odd, and we have

Lemma 3.2. For $k \geq 2, s>0$, and any primitive Dirichlet character,

$$
\begin{gathered}
\frac{q^{s} S_{\nu}(s, \chi)}{\tau(\chi)}=\sum_{j=1}^{k-1}(-1)^{j-1} F_{2 j} L(2 j, \bar{\chi})+\theta_{k} F_{2 k} \zeta(2 k), \quad \chi \text { even, } \\
\frac{q^{s} S_{\nu}(s, \chi)}{-i \tau(\chi)}=\sum_{j=1}^{k-1}(-1)^{j-1} F_{2 j-1} L(2 j-1, \bar{\chi})+\phi_{k} F_{2 k-1} \zeta(2 k-1), \quad \chi \text { odd },
\end{gathered}
$$

where $\left|\theta_{k}\right|<1$ and $\left|\phi_{k}\right|<1$.

Proof. For $\chi$ even, we have by the Fourier expansion of $f$ and (3.9),

$$
S_{\nu}(s, \chi)=\sum_{n=1}^{q-1} \frac{\chi(n)}{(q \nu+n)^{s}}=\frac{1}{q^{s}} \sum_{n=1}^{q-1} \chi(n) f\left(\frac{n}{q}\right)=\frac{\tau(\chi)}{q^{s}} \sum_{m=1}^{\infty} a_{m} \bar{\chi}(m),
$$

and (3.11) follows from summing (3.6) with $\theta_{k}=\sum \frac{(-1)^{k-1} \theta_{m, k} \overline{\chi(m)}}{m^{2 k}} / \zeta(2 k)$ which has the obvious bound. The odd case is similar.

Proof of Theorem 2.2. Choosing $k=2$ and summing over (3.11) for $\nu=t+1$ to $\infty$ using (3.5) gives

$$
E_{t+1}(s, \chi)=\frac{\tau(\chi)}{q^{s}}\left(\frac{s}{2 \pi^{2}} \frac{L(2, \bar{\chi})}{(t+1)^{s+1}}+\theta_{2} \frac{s(s+1)(s+2)}{8 \pi^{4}} \frac{\zeta(4)}{(t+1)^{s+3}}\right) .
$$

We now use the trivial bound $|L(2, \bar{\chi})| \leq \zeta(2)=\frac{\pi^{2}}{6}, \zeta(4)=\frac{\pi^{4}}{90}$ and the fact that $|\tau(\chi)|=\sqrt{q}$ in (3.13) to obtain (2.6). A similar estimate gives (2.7) in the odd case.

We also need the following

Lemma 3.3. For $\nu>5 / 4$ and $s \geq 3 / 2$, it holds that

$$
\frac{1}{\nu^{s}}-\frac{1}{(\nu+1)^{s}}>\frac{1}{\nu^{s+2}}-\frac{1}{(\nu+1)^{s+2}} \text {. }
$$


Proof. Since $\nu>1$, by multiplying both sides of (3.14) by $\nu^{s+2}(\nu+1)^{s+2}$, we see that it holds if and only if $(\nu+1)^{s+3}(\nu-1)>\nu^{s+3}(\nu+2)$ and clearly this will be true for all $s \geq 3 / 2$ if it is true at $s=3 / 2$. We need to prove that

$$
\left(\frac{\nu+1}{\nu}\right)^{9 / 2}>\frac{\nu+2}{\nu-1}
$$

or equivalently $f(x)=(2-x) x^{9 / 2}-(2 x-1)>0$, for $x=(\nu+1) / \nu \in(1,9 / 5)$, or that the polynomial $h(y)=f\left(y^{2}\right)=-y^{11}+2 y^{9}-2 y^{2}+1$ is positive for $y=\sqrt{x} \in$ $(1,3 / \sqrt{5})$ and this is true since it is easily shown that $h(1)=0, h^{\prime}(1)=3>0$ and the only positive roots of $h$ greater than 1 is around $1.35>3 / \sqrt{5}$.

Lemma 3.4. For any real nonprincipal character $\chi, L(2, \chi)>\frac{\pi^{2}}{15}$.

Proof.

$$
L(2, \chi)=\prod_{p \geq 2}\left(1-\chi(p) / p^{2}\right)^{-1}>\prod_{p \geq 2}\left(1+1 / p^{2}\right)^{-1}=\zeta(4) / \zeta(2)=\pi^{2} / 15 .
$$

3.3. Proof of Theorem $\mathbf{2 . 1}$ for even real primitive characters. We now assume $\chi$ is real primitive and even. In this case Gauss determined the sign of the Gauss sum so that $\tau(\chi)=\sqrt{q}$. For $1 / 2 \leq s \leq 1$, we pick $k=2$ in (3.11) and use $\zeta(4)=\pi^{4} / 90$ and (3.15) to obtain

$$
\frac{S_{\nu}(s, q) q^{s}}{\sqrt{q} s}>\frac{1}{30}\left(\frac{1}{\nu^{s+1}}-\frac{1}{(\nu+1)^{s+1}}\right)-\frac{1}{120}\left(\frac{1}{\nu^{s+3}}-\frac{1}{(\nu+1)^{s+3}}\right),
$$

and this gives (2.3) by setting $\nu=1$. Inequality (2.4) follows from Lemma 3.3.

\section{Estimates FOR SHIfTED CYCle SUMS}

4.1. Extension of a result of Leu and Li. In 1996, Leu and Li [5] generalized Davenport's result on $S_{v}(1, \chi)$ to shifted cycle sums. We shall now extend this to $1 / 2 \leq s \leq 1$. The main ideas are essentially those of Davenport but the details are more involved. Throughout this section, we assume $\chi$ is real, primitive and even. We define for $s>0$ and $\nu=0,1,2,3, \ldots$

$$
\begin{gathered}
A_{\nu}(s, \chi):=\sum_{n=\nu q+1}^{\nu q+\lfloor q / 2\rfloor} \frac{\chi(n)}{n^{s}}, \quad B_{\nu}(s, \chi):=\sum_{\substack{n=\nu q+\lfloor q / 2\rfloor+1 \\
n^{s}}}^{(\nu+1) q+\lfloor q / 2\rfloor} \frac{\chi(n)}{T_{\nu}(s, \chi)}:=B_{\nu}(s, \chi)+A_{\nu+1}(s, \chi)=\sum_{n=\nu q+\lfloor q / 2\rfloor+1}^{n^{s}} .
\end{gathered}
$$

Note that $S_{\nu}(s, \chi)=A_{\nu}(s, \chi)+B_{\nu}(s, \chi)$. The main estimate is the following generalization of Leu and Li's extension of Davenport's result to shifted sum:

Theorem 4.1. If $\chi$ is real, even and primitive, $\nu=0,1,2, \ldots, 1 / 2 \leq s \leq 1$, then

$$
T_{\nu}(s, \chi)<0 \text {. }
$$

Furthermore, for $\nu \geq 1$ we have more explicitly

$$
T_{\nu}(s, \chi)<\frac{s}{q^{s-1 / 2}} c(s)\left\{\frac{1}{(\nu+1 / 2)^{s+1}}-\frac{1}{(\nu+3 / 2)^{s+1}}\right\},
$$


where

$$
c(s)=\frac{(s+1)(s+2)}{720}-\frac{1}{32}<-\frac{11}{480} .
$$

The following corollary generalizing that in [5] is immediate.

Corollary 4.2. We have for any $t_{1}, t_{2} \geq 0$, and $1 / 2 \leq s \leq 1$,

$$
\begin{gathered}
\sum_{\nu=0}^{t_{1}} S_{\nu}(s, \chi)<L(s, \chi)<A_{0}(s, \chi)+\sum_{\nu=0}^{t_{2}} T_{\nu}(s, \chi), \\
A_{\nu}(s, \chi)>0 \text { for } \nu \geq 1, A_{0}(s, \chi)>L(s, \chi), \quad B_{\nu}(s, \chi)<0, \text { for } \nu \geq 0 .
\end{gathered}
$$

We will need the following

Lemma 4.3. For any real nonprincipal character $\chi$, we have

$$
H(\chi):=\sum_{m \geq 1} \frac{(-1)^{m} \chi(m)}{m^{2}}< \begin{cases}-\frac{\pi^{2}}{12} & \text { if } \chi(2) \in\{0,-1\} \\ -\frac{\pi^{2}}{16} & \text { if } \chi(2)=1\end{cases}
$$

Proof. We have

$$
H(\chi)=\left(-1+\frac{\chi(2)}{2^{2}}\right) \sum_{m o d d} \frac{\chi(m)}{m^{2}}=-\left(1-\frac{\chi(2)}{2^{2}}\right) \prod_{p \geq 3}\left(1-\frac{\chi(p)}{p^{2}}\right)^{-1},
$$

and the result follows from

$$
\prod_{p \geq 3}\left(1-\frac{\chi(p)}{p^{2}}\right)^{-1} \geq \prod_{p \geq 3}\left(1+\frac{1}{p^{2}}\right)^{-1}=\left(1+\frac{1}{2^{2}}\right) \frac{\zeta(4)}{\zeta(2)}=\frac{\pi^{2}}{12} .
$$

4.2. Proof of Theorem 4.1. We consider now the Fourier expansion of

$$
f(x)=\frac{1}{(\nu+x)^{s}} \sim \frac{a_{0}^{\prime}}{2}+\sum_{m=1}^{\infty}\left(a_{m}^{\prime} \cos 2 \pi m x+b_{m}^{\prime} \sin 2 \pi m x\right), \quad 1 / 2<x<3 / 2,
$$

Since $a_{m}^{\prime}=2 \int_{1 / 2}^{3 / 2} \frac{\cos 2 \pi m x}{(\nu+x)^{s}} d x$, we have as in (3.6) with $k=2$,

$$
\begin{aligned}
a_{m}^{\prime}= & (-1)^{m} \frac{2 s}{(2 \pi m)^{2}}\left\{\frac{1}{(\nu+1 / 2)^{s+1}}-\frac{1}{(\nu+3 / 2)^{s+1}}\right\} \\
& +(-1)^{m+1} \frac{2 s(s+1)(s+2)}{(2 \pi m)^{4}}\left\{\frac{1}{(\nu+1 / 2)^{s+3}}-\frac{1}{(\nu+3 / 2)^{s+3}}\right\} \\
& +\frac{2 s(s+1)(s+2)(s+3)}{(2 \pi m)^{4}} \int_{1 / 2}^{3 / 2} \frac{\cos 2 \pi m x}{(\nu+x)^{s+4}} d x .
\end{aligned}
$$

Since the amplitude in the last integral is decreasing, its sign must be that of $\cos 2 m(1 / 2) \pi=(-1)^{m}$. The mean value theorem gives

$$
\int_{1 / 2}^{3 / 2} \frac{\cos 2 \pi m x}{(\nu+x)^{s+4}} d x=(-1)^{m} \theta_{m}^{\prime} \int_{1 / 2}^{3 / 2} \frac{1}{(\nu+x)^{s+4}} d x
$$


where $0<\theta_{m}^{\prime}<1$. It follows that we have

$$
\begin{aligned}
a_{m}^{\prime}= & (-1)^{m} \frac{2 s}{(2 \pi m)^{2}}\left\{\frac{1}{(\nu+1 / 2)^{s+1}}-\frac{1}{(\nu+3 / 2)^{s+1}}\right\} \\
& +(-1)^{m+1} \frac{2 s(s+1)(s+2)}{(2 \pi m)^{4}}\left\{\frac{1}{(\nu+1 / 2)^{s+3}}-\frac{1}{(\nu+3 / 2)^{s+3}}\right\} \theta_{m, 2}^{\prime},
\end{aligned}
$$

where $0<\theta_{m, 2}^{\prime}=1-\theta_{m}^{\prime}<1$ depends on both $\nu$ and $s$.

By the Gauss sum (3.9), we get

$$
\begin{aligned}
& \quad \frac{q^{s} T_{\nu}(s, \chi)}{\sqrt{q}}=\frac{s}{2 \pi^{2}}\left\{\frac{1}{(\nu+1 / 2)^{s+1}}-\frac{1}{(\nu+3 / 2)^{s+1}}\right\} \sum_{m=1}^{\infty} \frac{(-1)^{m} \chi(m)}{m^{2}} \\
& +\frac{s(s+1)(s+2)}{8 \pi^{4}}\left\{\frac{1}{(\nu+1 / 2)^{s+3}}-\frac{1}{(\nu+3 / 2)^{s+3}}\right\} \sum_{m=1}^{\infty} \frac{(-1)^{m+1} \chi(m)}{m^{4}} \theta_{m, 2}^{\prime}(s) .
\end{aligned}
$$

If $\nu \geq 1$, the right-hand side can be bounded above using Lemma 4.3,

$$
\left|\sum_{m=1}^{\infty} \frac{(-1)^{m+1} \chi(m)}{m^{4}} \theta_{m, 2}^{\prime}(s)\right| \leq \zeta(4)=\pi^{4} / 90,
$$

and Lemma 3.3 which yields (4.4).

For $\nu=0$, we note that

$$
\frac{q^{s-1 / 2} T_{0}(s, \chi)}{s}=\frac{1}{2 \pi^{2}}\left(2^{s+1}-\frac{2^{s+1}}{3^{s+1}}\right) \sum_{m=1}^{\infty} \frac{(-1)^{m} \chi(m)\left(m^{2}-\lambda(s) \theta_{m, 2}^{\prime}(s)\right)}{m^{4}}
$$

where

$$
\lambda(s)=\frac{(s+1)(s+2)}{9 \pi^{2}}\left(\frac{3^{s+3}-1}{3^{s+1}-1}\right) .
$$

It thus suffices for us to show that

$$
Y(s):=\sum_{m=1}^{\infty} \frac{(-1)^{m} \chi(m)\left(m^{2}-\lambda(s) \theta_{m, 2}^{\prime}(s)\right)}{m^{4}}<0 .
$$

We need two more lemmas.

Lemma 4.4. For $1 / 2 \leq s \leq 1$, we have

$$
0.46044 \cdots=\frac{5(27 \sqrt{3}-1)}{12 \pi^{2}(3 \sqrt{3}-1)}=\lambda(1 / 2) \leq \lambda(s) \leq \lambda(1)=\frac{20}{3 \pi^{2}}=0.67547 \cdots .
$$

Proof. We set $s=S-1$ and $\mu(S)=S(S+1)\left(9 \cdot 3^{S}-1\right) /\left(3^{S}-1\right)=9 \pi^{2} \lambda(s)$, and we observe that $\mu^{\prime}(S)$ has the same sign as

$$
f(S)=(2 S+1)\left(9 \cdot 3^{S}-1\right)\left(3^{S}-1\right)-8 S(S+1) 3^{S} \log 3 .
$$

Since $3^{S}-1=e^{S \log 3}-1 \geq S \log 3$, we have

$$
\begin{aligned}
f(S) & \geq(S \log 3)\left((10 S+1) 3^{S}-(2 S+1)\right) \\
& \geq(S \log 3)((10 S+1)-(2 S+1)) \geq 8 S^{2} \log 3 \geq 0
\end{aligned}
$$

for $S \geq 0$. Hence $\lambda(s)$ increases with $s \geq-1$ and the results follow. 
As in [5] we will need to estimate $\theta_{1,2}^{\prime}$. From (4.10) with $\nu=0, m=1$, we have

$$
\theta_{1,2}^{\prime}(s)=1+\frac{s+3}{\left(2^{s+3}-\frac{2^{s+3}}{3^{s+3}}\right)} \int_{1 / 2}^{3 / 2} \frac{\cos 2 \pi x}{x^{s+4}} d x
$$

so that integrating by parts three times gives

$$
\lambda(s) \theta_{1,2}^{\prime}(s)=1-\frac{\pi 3^{s+1}}{2^{s}\left(3^{s+1}+1\right)} I(s),
$$

where

$$
I(s)=\int_{1 / 2}^{3 / 2} \frac{-\sin 2 \pi x}{x^{s+1}} d x .
$$

Lemma 4.5. For $1 / 2 \leq s \leq 1$, we have

$$
\lambda(s) \theta_{1,2}^{\prime}(s) \leq 1-\frac{9 \pi}{16} \int_{1 / 2}^{3 / 2} \frac{-\sin 2 \pi x}{x^{3 / 2}} d x=0.50590 \cdots .
$$

Proof. We split the integral into two parts and set $x=2-x$ in $\int_{1}^{3 / 2}$ to obtain

$$
I(s)=\int_{1 / 2}^{1}-\sin (2 \pi x) f(s, x) d x \text { with } f(s, x)=\frac{1}{x^{s+1}}-\frac{1}{(2-x)^{s+1}} .
$$

Since $x \leq 2-x$ for $x \leq 1$ and the function $g(x)=\frac{\log x}{x^{s+1}}$ is increasing for $s \geq$ $-1, x \leq 1$, the integrand in $I^{\prime}(s)$ is nonnegative and hence $I(s)$ is increasing. Since $\frac{\pi 3^{s+1}}{2^{s}\left(3^{s+1}-1\right)}$ decreases for $s>-1$, the result follows.

We are now ready to prove (4.15). Assume first (the easy case) where $\chi(2) \in$ $\{0,-1\}$. From the definition of $Y(s)$ in (4.15), (4.7) and Lemma 4.4 we have

$$
Y(s)<-\frac{\pi^{2}}{12}+\lambda(s) \zeta(4) \leq-\frac{\pi^{2}}{12}+\frac{20}{3 \pi^{2}} \frac{\pi^{4}}{90}=\frac{-\pi^{2}}{108}<0 .
$$

If $\chi(2)=1$, we consider the first three terms in (4.15)

$$
Y(s)=H(\chi)+\lambda(s) \theta_{1,2}^{\prime}(s)-\lambda(s) \frac{\theta_{2,2}^{\prime}(s)}{16}-\lambda(s) \sum_{m \geq 3} \frac{(-1)^{m} \chi(m) \theta_{m, 2}^{\prime}(s)}{m^{4}}
$$

and using (4.7), the fact that $\chi(2)>0, \lambda(s)>0,0<\theta_{i, 2}^{\prime}(s)<1$ and a trivial bound in the last term give

$$
\begin{aligned}
Y(s) & <-\frac{\pi^{2}}{16}+\lambda(s) \theta_{1,2}^{\prime}(s)+\lambda(s) \sum_{m \geq 3} \frac{1}{m^{4}} \\
& \leq-\frac{\pi^{2}}{16}+1-\frac{9 \pi}{16} \int_{1 / 2}^{3 / 2} \frac{\sin 2 \pi x}{x^{3 / 2}} d x+\frac{20}{3 \pi^{2}}\left(\frac{\pi^{4}}{90}-\frac{17}{16}\right) \\
& \approx-0.0975579 \cdots<0,
\end{aligned}
$$

where we used Lemma 4.5 in the last inequality. This completes the proof of Theorem 4.1. 


\section{NumericAl RESUltS}

We applied ALGO 1 to primitive even real characters of conductor $q$ up to 200,000 choosing the smallest $t$ for which $L_{t}(1 / 2, \chi)>0$ and using $\epsilon=0.00001$. It is found that the program always terminates with $s>1$ after finitely many steps and this proves Theorem 1.1. The computations for two simple cases $q=1085$ and 19032 are given in Figure 1 and Figure 2 where each plotted point corresponds to one iteration (one function and one derivative evaluation). The computations usually require a large number of iterations and take a long time to run. Statistics for the computations for the 46 exceptional primitive conductors $q$ up to 200,000 for which $S_{0}(s, \chi)<0$ is summarised in Table 1 . Each row gives the conductor $q$, the class number $h(q)$ of $Q(\sqrt{q})$, its regulator $R$, the negative values of $S_{0}(1 / 2, \chi)$, the smallest $t$ for which $L_{t}(1 / 2, \chi)>0$, the value of $L_{t}(s, \chi)$, the number of iterations, the clock time needed in seconds and finally the unique value $1 / 2<\alpha(q)<1$ where $S_{0}(s, \chi)$ crosses the zero line. The reader should be able to verify these computations independently by checking the number of iterations used. The timing of course depends on too many factors and is only meant as a guide of the relative amount of time needed. We have also proved numerically that $S_{0}^{\prime}(s, \chi)>0$ for the exceptional $q$ values at $1 / 2$ in Table 1 using Lemma 2.3 with $f(s)=S_{0}^{\prime}(s, \chi)$ and

$g(s)=\sum_{(n, q)=1, n=2}^{(t+1) q-1} \frac{(\log n)^{2}}{n^{s}}$ and a similar algorithm to ALGO 1. The computations can be done relatively fast as compared to the positivity of $L(s, \chi)$ because $S_{0}^{\prime}(s, \chi)$ is bounded further from zero because of the additional $\log n$ term. The value of $\alpha(q)$ is then determined by simple bisection.

We note that $S_{0}(1 / 2, \chi)<0$ tends to occur only for small class numbers though we have yet to formalise an exact relationship. The most time consuming case occurs for $q=142,637$ which requires 776,099 iterations over more than 5 days $\left(469,354\right.$ seconds) to run on our system. This is because $L_{t}(1 / 2, \chi)=0.00001$ is exceptionally small. We note that we can usually improve the performance by choosing a bigger $t$ than the least one which makes $L_{t}(1 / 2, \chi)>0$. For $q=142,637$, choosing $t=3$ in ALGO 1 requires only 121,254 iterations and runs more than 5 times faster (90175 seconds). Note that in this case, $L_{3}(0.5, \chi) \approx 0.00378$ is much closer to $L(0.5, \chi) \approx 0.00591$. It is clear we can always do better by choosing $t$ so that $L_{t}(0.5, \chi)>\alpha L(0.5, \chi)$ for some $\alpha>0.5$. Note that this can be done without computing $L(s, \chi)$ using $(2.6)$.

The algorithms are programmed in $\mathrm{C}$ with independent verification using PARIGP. The computations are done on a Linux PC cluster which comprises $8500-\mathrm{MHz}$ Pentium III PC with 256M RAM and total disk space of 8 GB, in the Department of Mathematics at the National University of Singapore. We applied the usual computational tricks. For example, since ALGO 1 is applied for a fixed $q$ at many different $s$, the values of $\log n$ are precomputed in double precision in a table and values of $n$ for which $\chi(n)$ is \pm 1 are remembered as indices. This is done only once and is then used at all the points where the function is evaluated. Note also that in most cases (e.g., when $Z / q^{*}$ is cyclic), $\chi(n)=1$ exactly for the quadratic residues which are found easily by taking squares up to $\lfloor q / 2\rfloor$ and using $\chi(n)=\chi(-n)$ without the need for the Euclidean algorithm for the Kronecker symbol. 
TABLe 1. Exceptional $q$ at $1 / 2$ up to 200,000: Time and iterations needed

\begin{tabular}{|c|c|c|c|c|c|c|c|c|}
\hline$q$ & $h(q)$ & $R$ & $S_{0}(0.5, \chi)$ & $t$ & $L_{t}(0.5, \chi)$ & Iterations & $\begin{array}{l}\text { Time } \\
(\mathrm{sec})\end{array}$ & $\alpha(q)$ \\
\hline 13340 & 4 & 6.1355 & -0.01645 & 3 & 0.00089 & 27509 & 1695 & 0.54909 \\
\hline 14693 & 1 & 18.001 & -0.01389 & 4 & 0.00025 & 179259 & 50523 & 0.55400 \\
\hline 16557 & 1 & 25.291 & -0.00912 & 1 & 0.00194 & 23269 & 2845 & 0.53545 \\
\hline 19032 & 4 & 8.7555 & -0.00477 & 1 & 0.00881 & 4373 & 61 & 0.51984 \\
\hline 37901 & 1 & $\begin{array}{l}35.072 \\
\end{array}$ & -0.01105 & 2 & 0.00208 & 71866 & 8738 & 0.53937 \\
\hline 42573 & 6 & 6.4281 & -0.00975 & 1 & 0.00101 & 52572 & 3185 & 0.53544 \\
\hline 50648 & 2 & 24.956 & -0.00830 & 1 & 0.00398 & 18831 & 5057 & 0.52860 \\
\hline 50853 & 4 & 11.211 & -0.00012 & 1 & 0.01072 & 13639 & 4266 & 0.50106 \\
\hline 53633 & 3 & 27.754 & -0.00382 & 1 & 0.01260 & 17585 & 9411 & 0.51296 \\
\hline 55037 & 5 & 7.0673 & -0.00861 & 1 & 0.00100 & 103674 & 60555 & 0.53643 \\
\hline 55733 & 1 & 32.339 & -0.00442 & 1 & 0.00503 & 46636 & 5712 & 0.52492 \\
\hline 60357 & 4 & 10.807 & -0.01284 & 2 & 0.00070 & 97518 & 11687 & 0.54249 \\
\hline 61333 & 1 & 63.313 & -0.00537 & 1 & 0.00683 & 31074 & 4227 & 0.51987 \\
\hline 61817 & 3 & 32.109 & -0.00507 & 1 & 0.01165 & 16448 & 5334 & 0.51494 \\
\hline 63416 & 1 & 71.246 & -0.00921 & 1 & 0.00407 & 2029 & 3047 & 0.52620 \\
\hline 68317 & 4 & 18.350 & -0.00168 & 1 & 0.01088 & 23487 & 5633 & 0.50844 \\
\hline 70949 & 1 & 58.183 & -0.00635 & 1 & 0.00441 & 46042 & 8621 & 0.52398 \\
\hline 89448 & 2 & 46.142 & -0.00257 & 1 & 0.01150 & 8607 & 1269 & 0.51049 \\
\hline 90461 & 1 & 62.809 & -0.01184 & 2 & 0.00161 & 105042 & 35418 & 0.53535 \\
\hline 91352 & 2 & 38.874 & -0.00351 & 1 & 0.00917 & 15236 & 8288 & 0.51441 \\
\hline 95717 & 1 & 38.805 & -0.00164 & 1 & 0.00772 & 50499 & 65342 & 0.51218 \\
\hline 96413 & 1 & 57.394 & -0.00035 & 1 & 0.00968 & 36022 & 35372 & 0.50288 \\
\hline 106917 & 6 & 10.481 & -0.00756 & 1 & 0.00316 & 48543 & 44436 & 0.52789 \\
\hline 109133 & 1 & 44.016 & -0.00482 & 1 & 0.00460 & 71691 & 15545 & 0.52577 \\
\hline 123653 & 1 & 52.612 & -0.00120 & 1 & 0.00835 & 50364 & 12393 & 0.50866 \\
\hline 125877 & 1 & 69.864 & -0.01420 & 3 & 0.00078 & 180915 & 264353 & 0.54003 \\
\hline 134357 & 4 & 13.180 & -0.00774 & 1 & 0.00179 & 121471 & 168781 & 0.53316 \\
\hline 137805 & 2 & 39.716 & -0.00966 & 1 & 0.00146 & 137805 & 66540 & 0.53007 \\
\hline 140460 & 4 & 30.441 & -0.00670 & 1 & 0.00758 & 10912 & 6151 & 0.51879 \\
\hline $142637 *$ & 5 & 11.667 & -0.00960 & 1 & 0.00001 & 776099* & 469354* & 0.53604 \\
\hline 144905 & 4 & 40.588 & -0.01391 & 1 & 0.00321 & 46556 & 71570 & 0.52510 \\
\hline 145517 & 3 & 23.139 & -0.00751 & 1 & 0.00248 & 100927 & 163109 & 0.52807 \\
\hline 147453 & 6 & 12.188 & -0.00605 & 1 & 0.00469 & 44888 & 38787 & 0.52391 \\
\hline 147773 & 3 & 16.689 & -0.00418 & 1 & 0.00523 & 79303 & 84223 & 0.52329 \\
\hline 159437 & 5 & 12.646 & -0.00297 & 1 & 0.00664 & 64293 & 77721 & 0.51627 \\
\hline 161192 & 2 & 46.526 & -0.00065 & 1 & 0.01163 & 19745 & 23023 & 0.50402 \\
\hline 161229 & $\overline{12}$ & 8.7033 & -0.01282 & 2 & 0.00207 & 86219 & 146494 & 0.53141 \\
\hline 170813 & 1 & 58.682 & -0.00187 & 1 & 0.00765 & 65035 & 95385 & 0.51228 \\
\hline 174605 & 6 & 14.352 & -0.00213 & 1 & 0.00823 & 41175 & 51431 & 0.51120 \\
\hline 176657 & 4 & 39.192 & -0.00613 & 1 & 0.01030 & 33230 & 48035 & 0.51607 \\
\hline 178485 & 4 & 22.371 & -0.00699 & 1 & 0.00412 & 41678 & 47460 & 0.52435 \\
\hline 179837 & 4 & 17.079 & -0.00079 & 1 & 0.00897 & 46694 & 103994 & 0.50582 \\
\hline 180197 & 1 & 66.343 & -0.00293 & 1 & 0.00663 & 68718 & 119778 & 0.51614 \\
\hline 192488 & 2 & 58.079 & -0.01242 & 1 & 0.00028 & 137823 & 128591 & 0.53013 \\
\hline 193973 & 4 & 14.228 & -0.00254 & 1 & 0.00687 & 70282 & 120195 & 0.51606 \\
\hline 199132 & 1 & 158.85 & -0.01131 & 1 & 0.00399 & 33981 & 48548 & 0.52414 \\
\hline
\end{tabular}




\section{ACKNOWLEDGMENTS}

We are greatly indebted to a very generous anonymous referee for very careful reading of the manuscript and many suggestions leading to much improved bounds in Theorem 2.1 and Theorem 4.1 and for ideas which led to much simpler proofs

of Lemma 3.3, Lemma 3.4 and all the lemmas in Section 4 as well as very useful suggestions which greatly improved our presentations.

\section{REFERENCES}

1. P. Bateman and E. Grosswald, On Epstein's zeta function, Acta Arith., 9 (1964) 365-373. MR 31:3392

2. J. B. Conrey and K. Soundararajan, Real zeros of quadratic Dirichlet L-functions, Invent. Math. 150 (2002) 1-44. MR 2004a:11089

3. H. Davenport, On the series for L(1), Journal of London Math. Soc., 24 (1949) 229-233. MR 11:162e

4. H. Davenport, Multiplicative Number Theory, Second Edition Revised by H. Montgomery, Graduate Texts in Mathematics, 74, Springer-Verlag, New York-Berlin (1980). MR 2001f:11001

5. M. G. Leu and W. Li, On the series for $L(1, \chi)$, Nagoya Math. J., Vol. 141 (1996), 125-142. MR 97c:11086

6. M. Low, Real zeros of the Dedekind zeta functions of an imaginary quadratic field, Acta. Arith., 14 (1968), 117-140. MR 38:4425

7. J. Rosser, Real zeros of real Dirichlet L-series, Bull. Amer. Math. Soc., 55, (1949) 906-913. MR 11:332c

8. M. Rubinstein, http://www.ma.utexas.edu/users/miker/L_function/VALUES/

9. P. Sarnak and A. Zaharescu, Some remarks on Landau-Siegel zeroes, Duke Math J., 111 (2002) no. 3, 495-507. MR 2002j:11097

10. A. Selberg and S. Chowla, On Epstein's Zeta-function, J. Reine Angew. Math. 227 (1967), 86-110. MR 35:6632

11. C. Siegel, Über die Classzahl quadratischer Zahlkörper, Acta. Arith. 1 (1935), 47-87.

12. K. Soundararajan, Nonvanishing of quadratic Dirichlet L-function at $s=1 / 2$, Ann. of Math. 152 (2000) no. 2, 447-488. MR 2001k:11164

13. M. Watkins, Real zeros of real odd Dirichlet $L$-functions, Math. Comp. 73 (2004), 415-423.

Software and Computing Programme, Institute of High Performance Computing, 1 Science Park Road, \#01-01, The Capricorn, Singapore Science Park II, Singapore 117528

E-mail address: chuaks@ihpc.a-star.edu.sg 